\title{
The Estimation of Physiologic Ability and Surgical Stress (E-PASS) model as a predictor of postoperative complications and mortality after digestive surgeries: a meta-analysis and systematic review
}

\author{
Guoqiao Chen ${ }^{1,2 \#}$, Yongle Chen ${ }^{1 *}$, Yili Dai ${ }^{1}$, Zhaoqi Shi ${ }^{1}$ Junhai Pan $^{1}$, Xiaoxiao Fan ${ }^{1,3}$, Hui Lin ${ }^{1,3}$ \\ ${ }^{1}$ Department of General Surgery, Sir Run Run Shaw Hospital, School of Medicine, Zhejiang University, Hangzhou, China; ${ }^{2}$ Department of \\ Emergency Medicine, Sir Run Run Shaw Hospital, School of Medicine, Zhejiang University, Hangzhou, China; ${ }^{3}$ Biomedical Research Center, Sir \\ Run Run Shaw Hospital, School of Medicine, Zhejiang University, Hangzhou, China \\ Contributions: (I) Conception and design: X Fan; (II) Administrative support: H Lin; (III) Provision of study materials or patients: G Chen, Y Chen; \\ (IV) Collection and assembly of data: Y Chen, Y Dai; (V) Data analysis and interpretation: G Chen, Z Shi; (VI) Manuscript writing: All authors; (VII) \\ Final approval of manuscript: All authors. \\ "These authors contributed equally to this work. \\ Correspondence to: Xiaoxiao Fan, PhD. Department of General Surgery, Sir Run Run Shaw Hospital, Zhejiang University School of Medicine, 3 East \\ Qingchun Rd, Hangzhou 310016, China. Email: fanxx_gs@zju.edu.cn; Hui Lin, PhD. Department of General Surgery, Sir Run Run Shaw Hospital, \\ Zhejiang University School of Medicine, 3 East Qingchun Rd, Hangzhou 310016, China. Email: 369369@zju.edu.cn.
}

\begin{abstract}
Background: Conflicting results exist about the usefulness of the Estimation of Physiologic Ability and Surgical Stress (E-PASS) model in patients undergoing digestive surgeries. Thus, this study aims to analyze and evaluate the E-PASS model for predicting postoperative complications and mortality.

Methods: A literature search strategy for "E-PASS" was carried out in PubMed, Embase, Google Scholar, Ovid, and Cochrane databases for studies published before November 2020. "E-PASS" or "Estimation of Physiologic Ability and Surgical Stress" were used as the search terms in all databases, and a total of 20 published English language studies with available data relating to digestive surgery were selected for this study. Clinical data and indices including preoperative risk score (PRS), surgical stress score (SSS), comprehensive risk score (CRS), postoperative complications, mortality, and overall survival (OS) were collected. Meta-analyses of heterogeneity were performed using Review Manager version 5.3 and STATA 14.0.

Results: Twenty studies with 9,136 patients were included in our meta-analysis. Using a random-effects model, the indices of the E-PASS model in patients with postoperative complications were significantly greater than those in patients without complications [PRS: 95\% confidence interval (CI): 0.14-1.50; SSS: 95\% CI: 0.28-1.06; CRS: 95\% CI: 048-1.49]. Moreover, patient morbidity and mortality were associated with a higher CRS (morbidity: 95\% CI: 2.17-6.29; mortality: 95\% CI: 1.57-4.78), while subgroup analyses showed that a high CRS in the elderly was related to increased morbidity (95\% CI: 1.57-4.78) without heterogeneity. In addition, we found that a high E-PASS CRS was significantly associated with shorter OS after digestive surgery (95\% CI: 1.24-5.41).

Discussion: The higher CRS score in the E-PASS model accompanies with many postoperative complications, increased mortality and shorter OS. Collectively, the E-PASS model is a convenient and effective risk assessment for patients undergoing digestive surgeries. More stringently designed studies are expected to develop better estimates of the application value of this model in digestive surgeries.
\end{abstract}

Keywords: Estimation of physiologic ability and surgical stress; comprehensive risk score; digestive surgeries; meta-analysis

Submitted Apr 18, 2021. Accepted for publication Jul 20, 2021.

doi: $10.21037 / \mathrm{apm}-21-941$

View this article at: https://dx.doi.org/10.21037/apm-21-941

\footnotetext{
^ ORCID: 0000-0003-4898-9535.
} 


\section{Introduction}

Many complex procedures, such as gastrectomy, radical operation for colon cancer, liver resection, pancreatectomy, and pancreaticoduodenectomy, fall under the heading of digestive surgeries. Severe complications and relatively high mortality often accompany major digestive surgery. For example, the pancreaticoduodenectomy shows an incidence of complications as high as $60 \%$ and mortality of about $4 \%$ (1). The incidence of postoperative complications for hepatectomy was about $29.4 \%$, and the mortality was $5.2 \%$ (2), while for gastrectomy, the morbidity ranged from $20 \%$ to $46 \%(3,4)$. Postoperative complications are associated with increased length of hospitalization, high costs, and poor prognoses. Thus, an accurate risk assessment would assist surgeons in making correct decisions and design individually-focused therapeutic strategies.

The Estimation of Physiologic Ability and Surgical Stress (E-PASS) model was proposed by Haga et al. (5) in 1999, in a study that aimed to predict gastrointestinal surgical risks. The E-PASS model consists of 3 indicators, namely the preoperative risk score (PRS), the surgical stress score (SSS), and the comprehensive risk score (CRS), and considers 10 variables. Seven of the ten variables relate to physiologic factors of patients, while the remainder relates to operative factors. The PRS contains the following 6 variables: age $\left(\mathrm{X}_{1}\right)$; the severity of heart disease (presence or not, $\mathrm{X}_{2}$ ); the severity of pulmonary disease (presence or not, $\mathrm{X}_{3}$ ); diabetes mellitus (presence or not, $\mathrm{X}_{4}$ ); the Eastern Cooperative Oncology Group performance status index (ECOG-PS, range, 0-4, $\mathrm{X}_{5}$ ) (6); and the American Society of Anesthesiologists physiologic status classification (ASA, range, $\left.1-5, X_{6}\right)(7)$. The equation for PRS is as follows: PRS $=-0.0686+0.00345 X_{1}+0.323 X_{2}+0.205 X_{3}+0.153 X_{4}$ $+0.148 X_{5}+0.0666 X_{6}$. Four variables are included in the SSS: blood loss divided by body weight (gram/kilogram, $\mathrm{X}_{7}$ ); the operative time in hours $\left(\mathrm{X}_{8}\right)$; and the extent of the skin incision (range, $0-2, X_{9}$ ). The equation of SSS is: SSS $=-0.342+0.0139 X_{7}+0.0392 X_{8}+0.352 X_{9}$. The CRS is calculated using the PRS and the SSS, with the formula of the CRS as follows: $\mathrm{CRS}=-0.328+0.936 \times \mathrm{PRS}+0.976 \times$ SSS.

To date, conflicting results exist about the predictive value of the E-PASS model for digestive surgeries. Our study aims to conduct a meta-analysis to assess the application value and availability of the E-PASS model for predicting postoperative complications and mortality in patients undergoing digestive surgeries.
We present the following article in accordance with the PRISMA reporting checklist (available at https://dx.doi. org/10.21037/apm-21-941).

\section{Methods}

\section{Search strategy}

We searched the PubMed, Embase, Google Scholar, Ovid, and Cochrane databases for studies published before November 2020. The terms used for the final search in all databases were "E-PASS" or "Estimation of Physiologic Ability and Surgical Stress." Titles and abstracts were used for preliminary screening, and full texts were then analyzed. The references of the identified publications were also examined to obtain additional studies. These publications gave us a selection of 20 English language studies with available data relating to digestive surgery.

\section{Inclusion and exclusion criteria}

The inclusion criteria were as follows: study type (all casecontrol studies); participants (patients who underwent digestive surgery); comparison (any study that compared the E-PASS model between the patients with complications and without complications, or any study that compared the incidence of morbidity and mortality between a high E-PASS CRS group and a low E-PASS CRS group); outcomes (any form of results we were interested in, such as postoperative complications, morbidity, mortality, and overall survival). The postoperative complications expressly referred to any levels of complications classified as ClavienDindo, because some studies had unspecified descriptions, while some studies had Clavien-Dindo II/III/IV/V complications (8-17).

The following exclusion criteria were applied: studies without a control group (case series, case reports); data not involving digestive surgeries; reviews; articles that could not provide comparative outcomes; articles concerning a modified E-PASS; literature written in a language other than English.

\section{Data extraction and quality assessment}

Extracted data included the first author, publication year, country of origin, surgical site, journal name, patient age and gender, CRS cut-off, the area under the receiver operative characteristic curve (AUC), postoperative 
complications, mortality, the E-PASS scores (PRS, SSS, and CRS), and the overall survival (OS) data. The specific digestive surgeries in these studies referred to hepatobiliary-pancreatic (HBP) and gastrointestinal (GI) surgeries. The OS was defined as the interval between the surgical treatment and the death of a patient or their last follow-up. The hazard ratio (HR) and 95\% confidence intervals (95\% CIs) were obtained directly from each article.

The quality of the non-randomized trials was critically assessed according to the Newcastle-Ottawa Scale (NOS) (18). A NOS score $<6$ was considered indicative of moderate or low quality. Any discrepancy was resolved by consultation with all authors.

\section{Statistical analysis}

A meta-analysis was performed according to the PRISMA Statement. Both dichotomous and continuous variables were analyzed using the inverse variance method, with the odds ratio (OR) and 95\% CI calculated for the dichotomous outcomes. The standardized mean differences (SMDs) and $95 \%$ CIs were calculated for the continuous data, and the $\mathrm{I}^{2}$ and $\mathrm{P}$ statistics were used to test heterogeneity. An $\mathrm{I}^{2}<50 \%$ and $\mathrm{P}>0.05$ were considered indicative of low or no heterogeneity, and because of this, a fixed-effects model was used. Otherwise, a random-effects model was used. The Begg's and Egger's tests were used to assess any publication bias.

Subgroup analysis and meta-regression were performed to explore the potential causes of the heterogeneity. In the subgroup analyses, both the random-effects and fixedeffects models were performed to generate the pooled ORs. Review Manager version 5.3 and STATA 14.0 were used to perform the meta-analysis.

\section{Results}

\section{The screening and characteristics of the included studies}

The flowchart of our systematic literature search is presented in Figure 1. Two hundred and twenty-five records were initially identified by exploring 5 databases (PubMed, Embase, Google Scholar, Ovid, and Cochrane Database) and other sources. After removing duplicate publications, we screened 178 records for further assessment. We obtained 44 articles by scanning titles and abstracts, of which 36 records were excluded because of the unavailability of the full text. Moreover, 24 full-text articles were excluded due to their language, inclusion of invalid data, or use of a different model. Ultimately, 20 studies were included in our quantitative analysis.

The main characteristics of the included studies in our meta-analysis are shown in Table 1. The included 20 studies featured 9,136 patients, of which 17 studies were from Japan, and the remaining 3 studies were from Switzerland and Russia. As for surgical type, 12 studies discussed the GI region, and 8 studies discussed the HBP region. Additionally, different age categories were observed, with 5 studies focusing on the elderly. The chosen studies also defined different CRS cut-off values, of which 10 studies used a value of 0.5 . The reported AUC values ranged from 0.542 to 0.890 , and according to the NOS scores, the quality assessments for all studies were of moderate to high quality (NOS $\geq 6$ ).

\section{The differences between PRS, SSS and CRS in patients with and without postoperative complications after digestive surgery}

In the analysis of PRS, a total of 1,076 patients were enrolled in the six studies $(11,15,19-22)$. After conducting pooled analysis using a random-effects model $\left(\mathrm{I}^{2}=96 \%\right.$, $\mathrm{P}<0.001$, Figure $2 A ; \mathrm{I}^{2}=97 \%, \mathrm{P}<0.001$, Figure $\mathrm{S} 1$ ), we found that the PRS in patients with postoperative complications was significantly greater than it in the patients without complications (SMD $=0.82,95 \%$ CI: $0.14-1.50, \mathrm{P}=0.020$, Figure $2 A$; $\mathrm{SMD}=1.77,95 \% \mathrm{CI}: 0.26-3.27, \mathrm{P}=0.020$, Figure S1).

As for the SSS, the collected data from the above studies were analyzed, with a random-effects model performed because of the high heterogeneity among the articles $\left(\mathrm{I}^{2}=87 \%, \mathrm{P}<0.001\right.$, Figure $\left.2 B\right)$. The patients with complications after surgery had significantly high SSS scores (SMD $=0.67,95 \%$ CI: $0.28-1.06, \mathrm{P}<0.001$, Figure 2B).

Included were 8 studies with 1,197 patients that explored the differences in the CRS $(11,12,15,19-23)$. High heterogeneity was found across all studies, with a random-effects model being applied $\left(\mathrm{I}^{2}=93 \%, \mathrm{P}<0.001\right.$, Figure 2C). The presence of complications was associated with a greater CRS when compared to the CRS in patients without postoperative complications ( $\mathrm{SMD}=0.99,95 \% \mathrm{CI}$ : 048-1.49, $\mathrm{P}<0.001$, Figure 2C).

The subgroup analyses we conducted to explore the heterogeneity sources of the PRS, the SSS, and the CRS included: sample size $(\geq 200$ "versus" $<200)$; location 

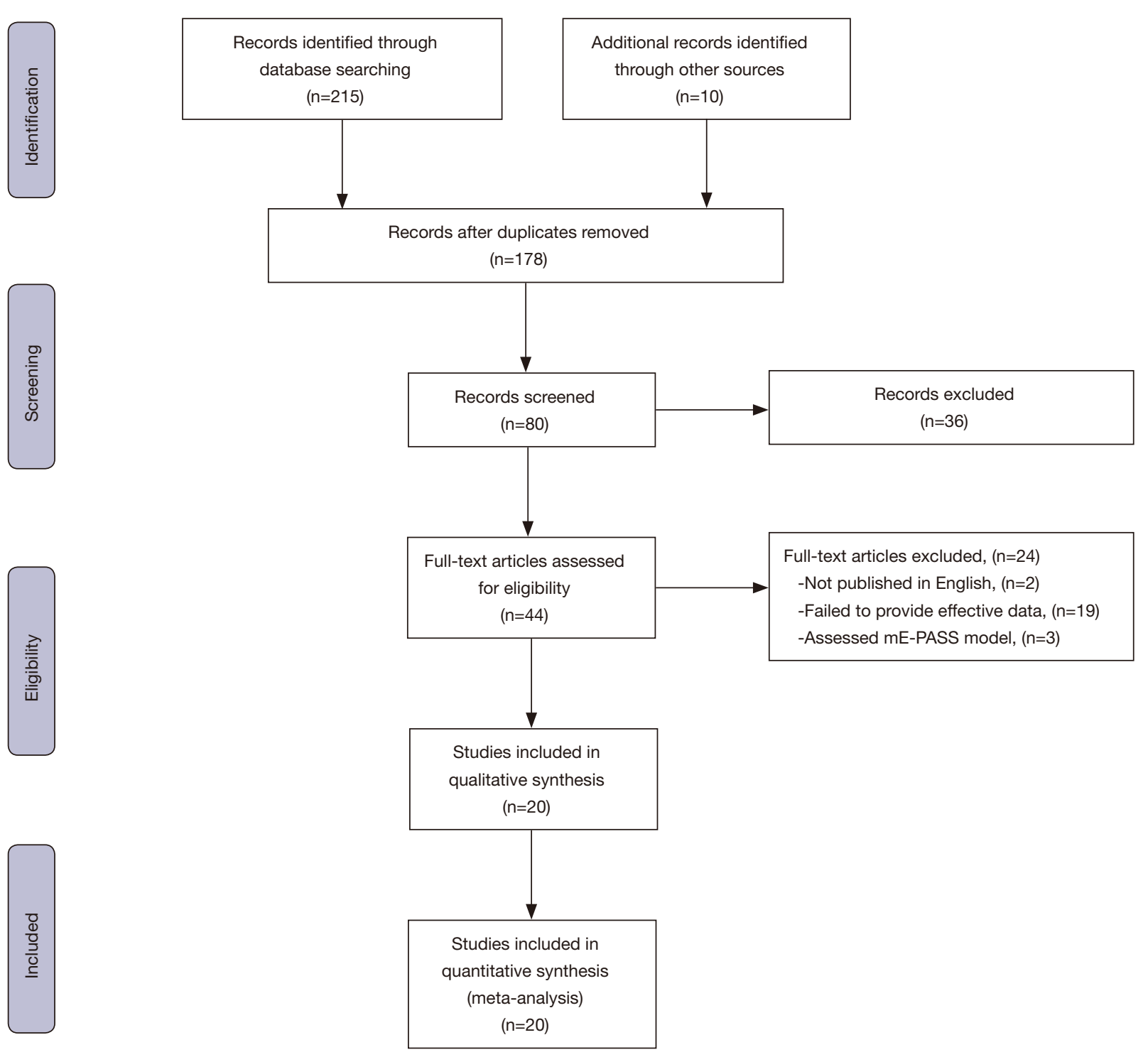

Figure 1 Flowchart of identified and included studies.

(Japan "versus" not Japan), surgery (GI surgery "versus" HBP surgery); age (elderly "versus" younger); and CRS cut-off value ( 0.5 "versus" not 0.5$)$. In the PRS, most of these subgroups were not significant in patients with and without postoperative complications except for 2 subgroups (population from Japan: $\mathrm{P}=0.027$ and CRS cut-off not equal to 0.5: $\mathrm{P}=0.022$, Table $\mathrm{S} 1$ ). As for the SSS and the CRS, significant differences were still present in all subgroups except for the analyses whose samples sizes were $\geq 200$ $(\mathrm{P}=0.056$, Table S1).

The 3 analyses of publication bias were not found in these studies (PRS: $\operatorname{Pr}>|Z|=0.133$, SSS: $\operatorname{Pr}>|Z|=0.060$,
CRS: $\operatorname{Pr}>|Z|=0.063$, Figure S2A-S2C).

The comparison of morbidity or mortality between high and low CRS group

We further compared the morbidity in the 2 CRS groups, analyzing 15 studies that included 2,266 digestive surgery patients $(1,9,10,13-17,19-25)$. With regard to heterogeneity $\left(\mathrm{I}^{2}=81 \%, \mathrm{P}<0.001\right.$, Figure $\left.3 A\right)$, the pooled data demonstrated that patients with a high CRS were expected to have higher chance of morbidity (OR $=3.69,95 \%$ CI: $2.17-6.29$, $\mathrm{P}<0.001$, Figure $3 A$ ). No publication bias was found in 


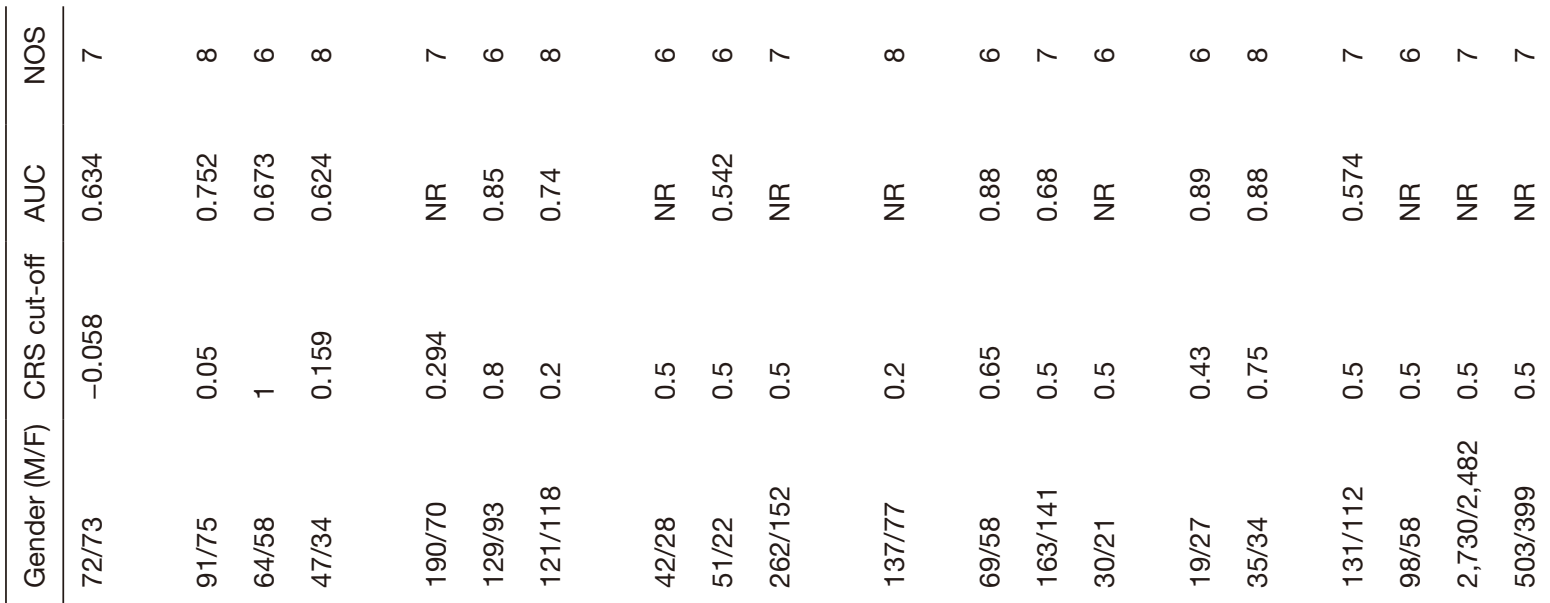

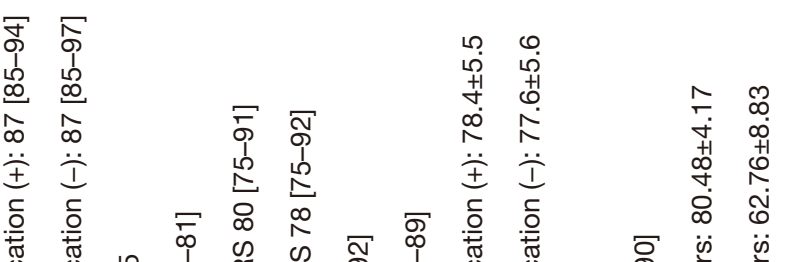

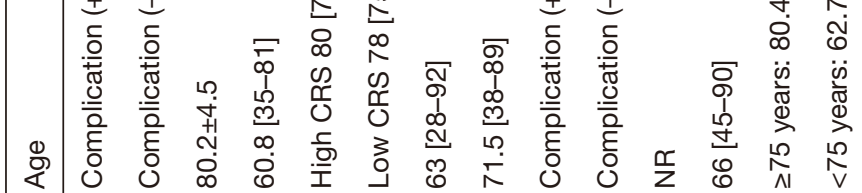

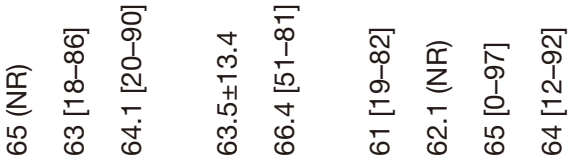

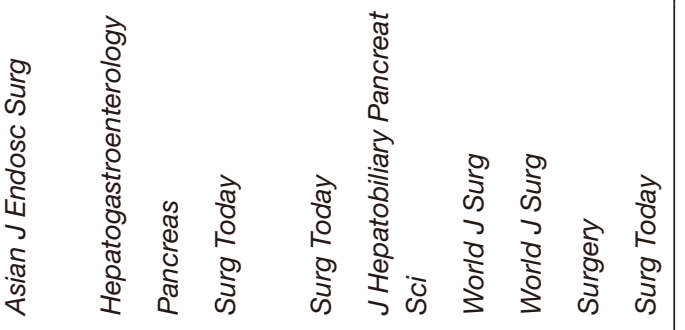

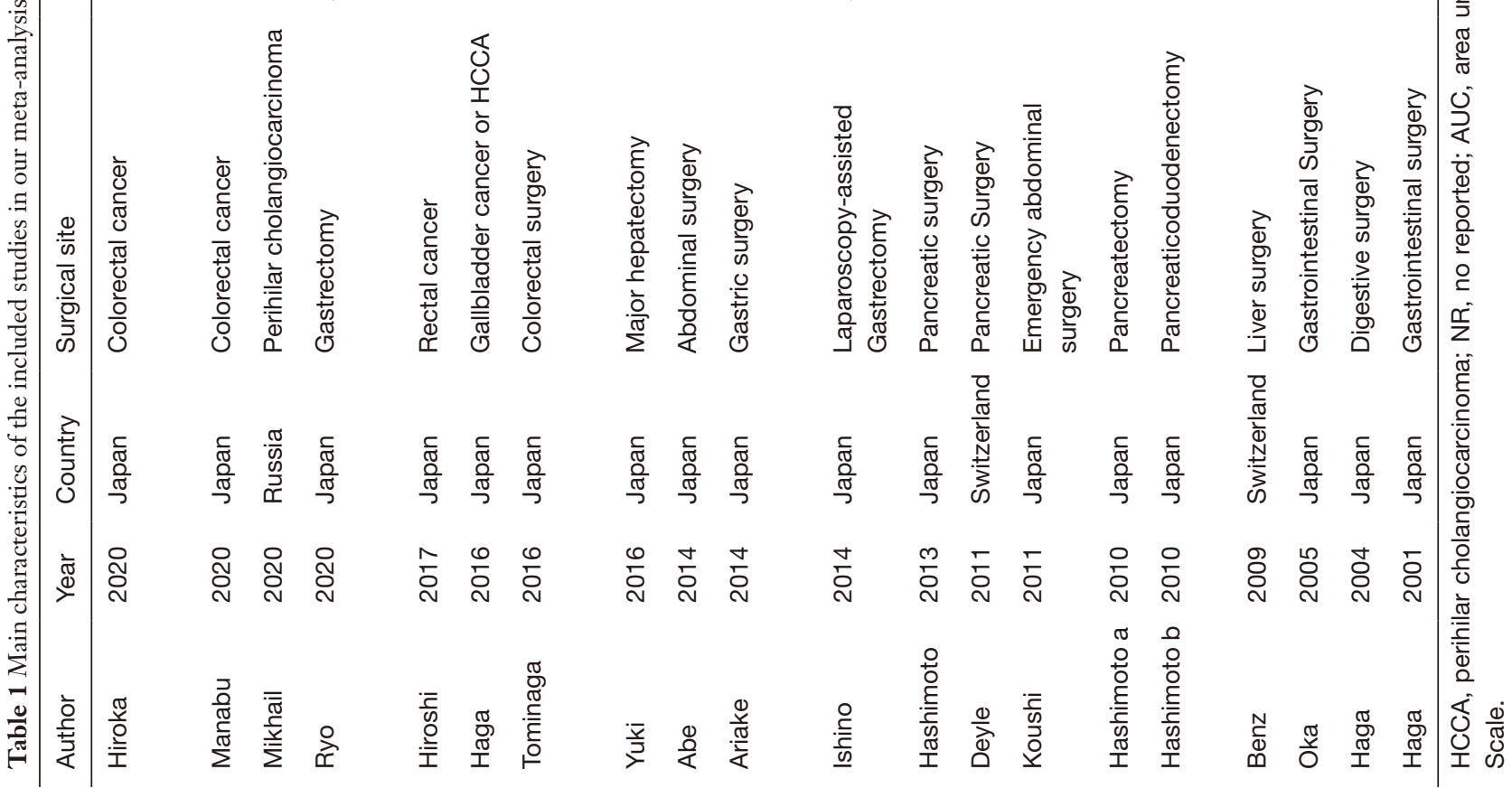


A

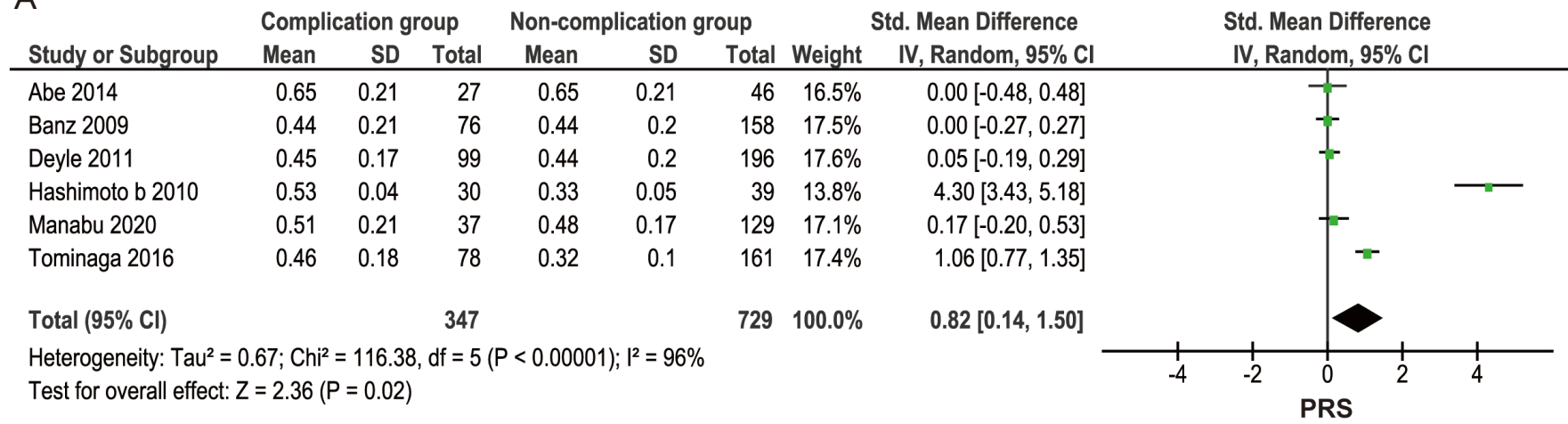

B

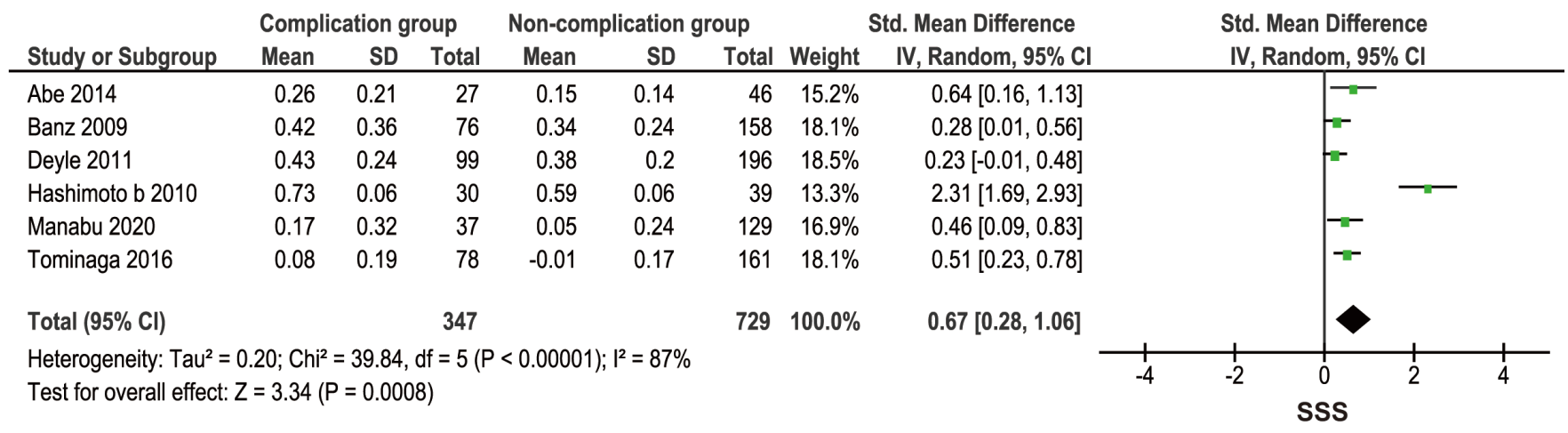

C

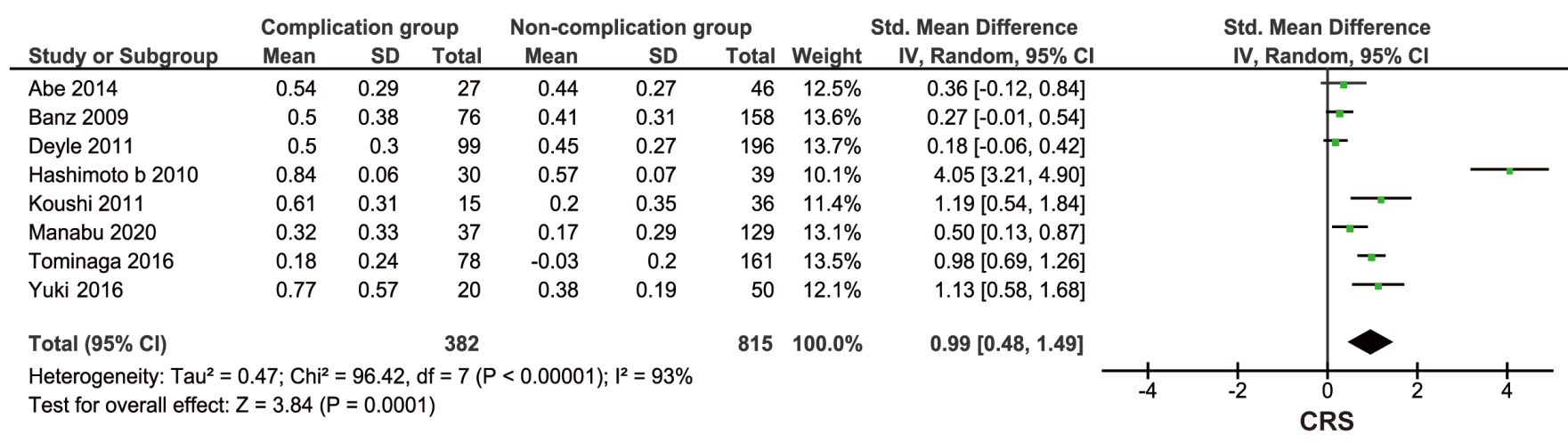

Figure 2 Forest plot of comparison: the group with postoperative complications $v s$. the group without complications. (A) The value of the PRS; (B) the value of the SSS; (C) the value of the CRS. PRS, preoperative risk score; SSS, surgical stress score; CRS, comprehensive risk score.

our research $(\operatorname{Pr}>|Z|=0.428$ for Begg's test, Figure S2D; $\mathrm{P}=0.690$ for Egger's test).

To explore the source of heterogeneity, we conducted subgroup and meta-regression analyses. According to the sample size ( $\geq 200$ "versus" <200), location (Japan "versus" not Japan), surgery (GI surgery “versus” HBP surgery), age (elderly "versus" younger), and CRS cut-off value (0.5 "versus" not 0.5$)$, the majority of subgroup analyses achieved a similar prognostic result to the CRS, except for small sample size $(\mathrm{P}=0.051)$ and non-Japanese reports 
A

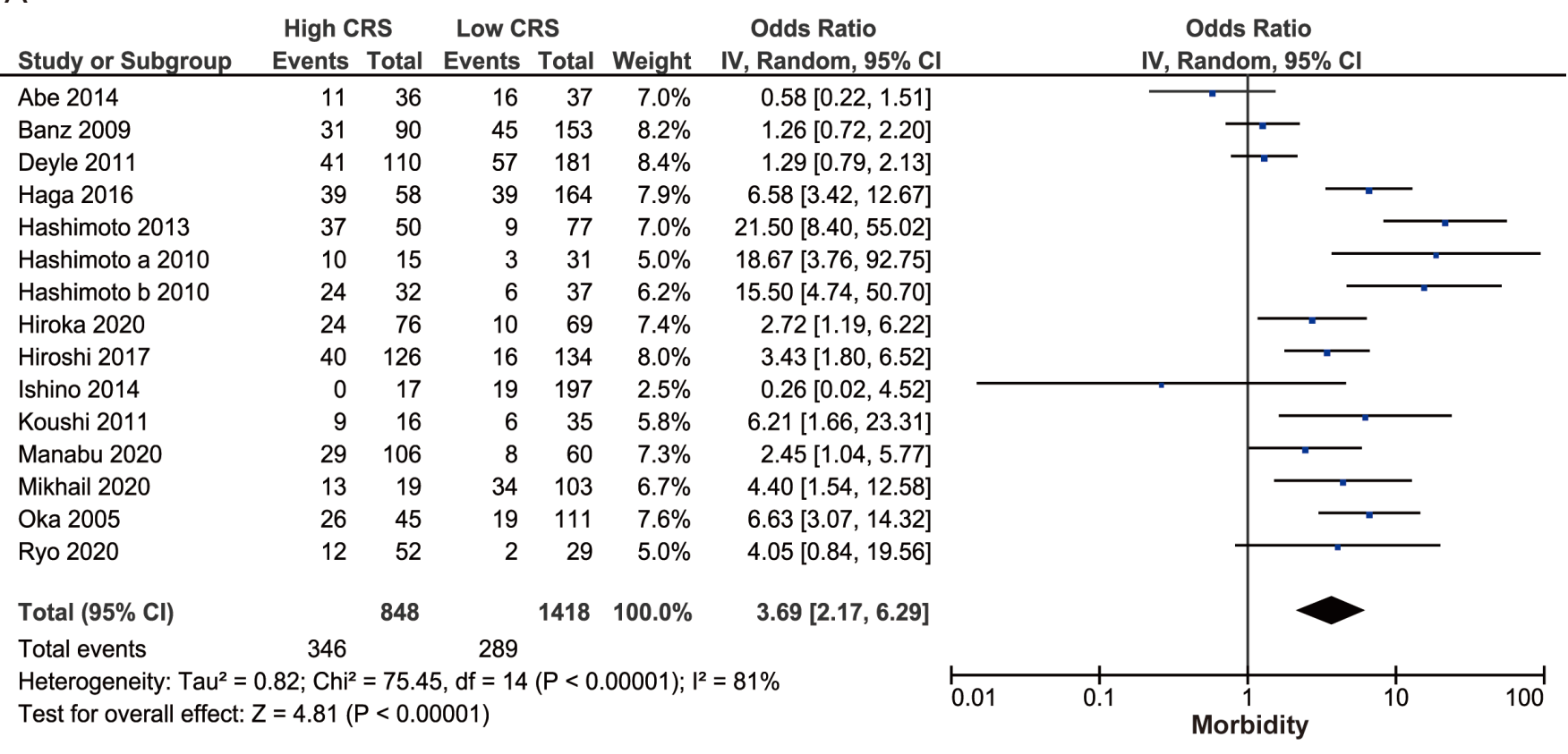

B

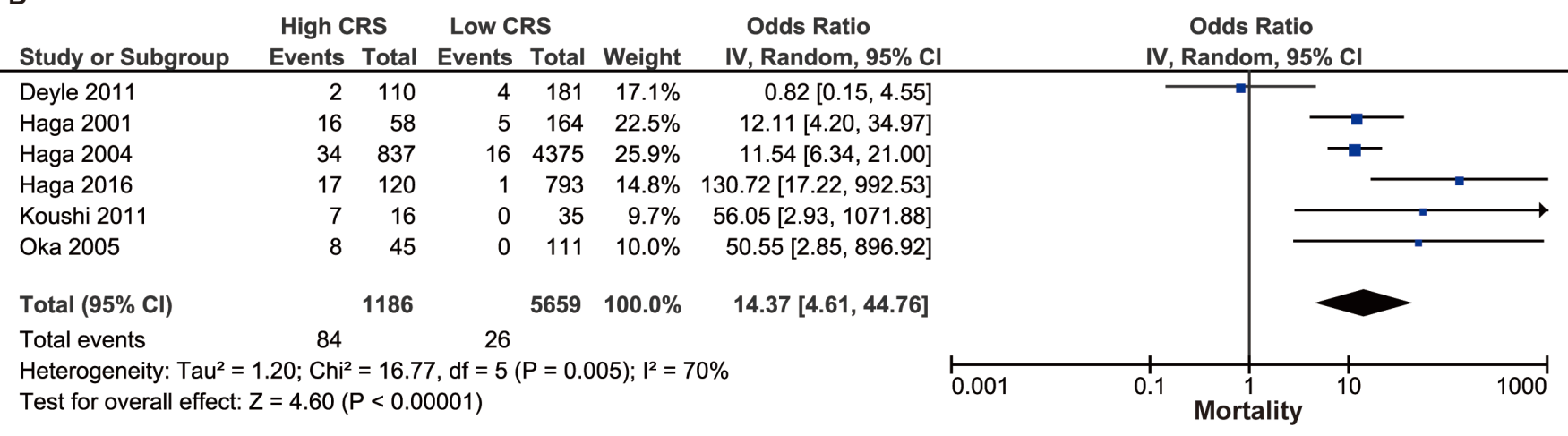

Figure 3 Forest plot of comparison: the high E-PASS CRS group vs. the low E-PASS CRS group. (A) postoperative morbidity; (B) mortality. E-PASS, Estimation of physiologic ability and surgical stress; PRS, preoperative risk score; SSS, surgical stress score; CRS, comprehensive risk score.

$(\mathrm{P}=0.100)$ (Table 2). In addition, the elderly subgroup shown in the high CRS group was related to higher morbidity (OR $=2.74,95 \%$ CI: $1.57-4.78, \mathrm{P}<0.001)$ without heterogeneity $\left(\mathrm{I}^{2}=0, \mathrm{P}=0.86\right)$. As for meta-regression analysis, the CRS cut-off value might partially explain heterogeneity source $(\mathrm{P}=0.089)$.

Meanwhile, we compared the mortality between the high and low CRS groups and included 6 articles with 6,845 patients $(10,21,23,25-27)$. The pooled analysis (OR $=14.37$, 95\% CI: 4.61-44.76, $\mathrm{P}<0.001$, Figure $3 B$ ) showed an obvious relationship between a high CRS and higher mortality with heterogeneity $\left(\mathrm{I}^{2}=70 \%, \mathrm{P}=0.005\right.$, Figure $\left.3 B\right)$.
The funnel plot shows the rough symmetry determined by Begg's test $(\operatorname{Pr}>|Z|=0.260$, Figure $S 2 E)$, while the $P$ value of Egger's test was 0.077 .

\section{The relationship between overall survival and high CRS}

Four studies presented follow-up information and 1,033 patients were enrolled $(8,9,11,15)$, but no heterogeneity was detected $\left(I^{2}=0, P=0.839\right.$, Figure 4$)$. The pooled estimates showed that a high E-PASS CRS was significantly associated with shorter OS after digestive surgery (HR $=3.32,95 \%$ CI: $1.24-5.41, \mathrm{P}=0.002$ ). The funnel plot shows 
Table 2 Summary of subgroup and meta-regression analyses for morbidity in high and low CRS groups

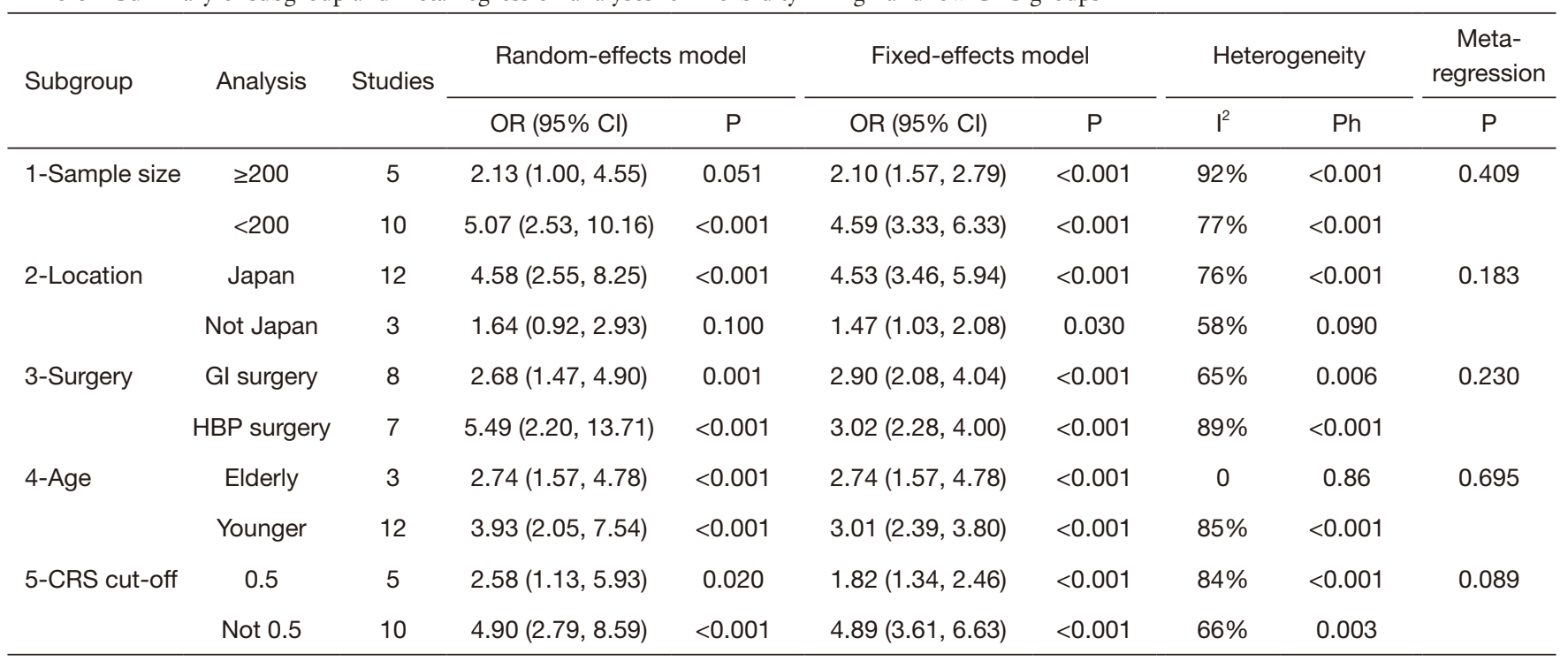

OR, odds ratio; $\mathrm{Cl}$, confidence interval; $\mathrm{Ph}, \mathrm{P}$ value of $\mathrm{Q}$ test for heterogeneity test; GI, gastrointestinal; HBP, hepato-biliary-pancreatic; CRS, comprehensive risk score.

\begin{tabular}{|c|c|c|}
\hline Author name (publication year) & ES $(95 \% \mathrm{Cl})$ & Weight (\%) \\
\hline Tominaga (2016) & $5.33(2.18,13.10)$ & 14.57 \\
\hline Ishino (2014) & $1.64(0.19,14.00)$ & 9.11 \\
\hline Ariake (2014) & $3.83(1.22,12.40)$ & 13.90 \\
\hline Manabu (2020) & $2.99(1.35,6.62)$ & 62.42 \\
\hline Overall $(I-$ squared $=0.0 \%, p=0.839)$ & $3.32(1.24,5.41)$ & 100.00 \\
\hline$Z=3.13 \quad p=0.002$ & & \\
\hline-14 & 14 & \\
\hline
\end{tabular}

Figure 4 Forest plot compares OS between the high E-PASS CRS group and the low E-PASS CRS group. OS, overall survival; E-PASS, Estimation of physiologic ability and surgical stress; CRS, comprehensive risk score.

the approximately symmetry $(\operatorname{Pr}>|Z|=0.734$ for Begg's test, Figure S2F; $\mathrm{P}=0.113$ Egger's test).

\section{Discussion}

The E-PASS was first proposed by Haga et al. in 1999 (5), and it took into account both preoperative and surgical factors. To date, our study is the first meta-analysis and systematic review to discuss the efficiency of the E-PASS model in predicting postoperative complications and mortality in patients undergoing digestive surgeries.

Our pooled analyses showed that 3 indicators (PRS, SSS, CRS) from the E-PASS model were associated with current postoperative complications in patients after digestive surgeries. We conducted 2 pooled analyses to estimate the preoperative effect of the PRS on the 3 included studies with an SMD equal to 0, but we acquired the same result. Meanwhile, the 5 subgroup analyses, including sample size, location, surgery, age, and CRS cut-off for those indicators, demonstrated that the SSS or the CRS had significant 
predictive abilities when applied to these subgroups, but the PRS was not significant in most subgroups. The PRS as a predictive factor was used to evaluate the patient's physiologic status, but it showed that the stress of digestive surgery plays a major role in this prediction. The CRS calculated by the PRS and the SSS has comprehensive and accurate prediction abilities relating to postoperative complications in patients after digestive surgeries, as Hirose et al. (28) reported in a multicenter study on patients with hip fractures. Moreover, some studies also demonstrated the ability of the E-PASS model to predict certain complications, such as liver failure (29) and anastomotic leakage (30). Furthermore, our study revealed that the incidence of morbidity and mortality following digestive surgeries was higher in the high CRS group when compared with the low CRS group, which confirms and strengthens the qualities of the CRS.

To date, many prognostic systems have been proposed as predictors of prognosis following major surgeries. The Glasgow score (31), the neutrophil-to-lymphocyte ratio (32), and the prognostic nutritional index (PNI) (33) are currently 3 of the most widely-used models. These 3 models were proven to be effective for predicting survival and complications in patients who have had various kinds of digestive surgeries (34-36). Our meta-analysis not only showed that a high E-PASS CRS was also associated with poor prognosis (low OS) after digestive surgeries, but it also demonstrated the relationship between a high E-PASS CRS and postoperative complications in patients after digestive surgeries. However, we paid close attention to the postoperative complications, and we were unable to make a definite conclusion due to the limited number of studies that discussed survival. We hope that future studies are encouraged to explore this potential connection. Furthermore, the relationship between the tumor differentiation grade of the tumor, node, and metastasis (TNM) stage and the E-PASS system in patients with cancer is also worth exploring.

Unfortunately, significant heterogeneity was observed across the included studies, which might cast doubt on our conclusions' reliability. This heterogeneity might exist in terms of patient demographics, study design, type of surgery, the duration of follow-ups, and different CRS cutoff values. A difference in patient age was also considered a reason for the heterogeneity source in our pooled analysis. Older adults are generally considered to have poor physical fitness and reduced recovery capacity after surgery. The E-PASS model was more inclined to show a high CRS score in the elderly. Some studies paid attention to the idea that the E-PASS model was more effective for the elderly $(14-16,37)$ than for all ages or specifically younger people. The different CRS cut-off values might be a source of heterogeneity according to the results of the meta-regression. Some studies' critical CRS cut-off value (19-21,23-25) was 0.5 , but those studies were all conducted in the previous decade and did not compare age differences. Due to recent economic, radiological technology, and genetic diagnostic developments, the cut-off value in recent studies might be smaller because of early diagnoses. In addition, the evolution of surgery techniques such as laparoscopic surgery has decreased both surgical time and hemorrhage volume. As these factors directly or indirectly changed the PRS or the SSS, the CRS cut-off value would show a considerable alteration. Regrettably, we could not account for these differences due to a lack of detailed information, despite using appropriate meta-analytic methods with random-effects models.

The AUC values of the included studies varied from each other, with 6 studies reporting that the E-PASS model did not accurately predict morbidity, showing AUC values $<0.7$ (14,16,17,19-21). Furthermore, the E-PASS model lost its accuracy in the western population, as demonstrated by the 3 studies from western countries in our pooled analyses which reported low AUC values (17,20,21). Besides, the subgroup analysis of non-Japanese locations in our study showed no significance in comparing postoperative morbidity between the high and low CRS groups $(\mathrm{P}=0.010)$. In the studies from Japan, the E-PASS model showed strong predictive power, with 4 Japanese articles reporting that the AUC value was higher than $0.85(1,10,22,24)$. Confusingly, it is reported that E-PASS CRS of older people after abdominal surgery was a useful assessment for mortality but not for postoperative morbidity (AUC <0.5) (36). However, the small sample size of 201 patients in that study might be the cause of the difference, but further research needs to be done to answer this question.

The Physiological and Operative Severity Score for the enUmeration of Mortality and morbidity (POSSUM) model, which was proposed by Copeland et al. (6), is most widely used for predicting postoperative morbidity and mortality following digestive surgeries. Many studies had proven it to be an effective system, but in contrast to the Copeland study, several studies have pointed out that this system might overestimate mortality and morbidity rates $(27,38)$. It has been reported in some studies that the E-PASS model showed better predictive capabilities than 
the POSSUM model (11). In addition, a modified E-PASS model (mE-PASS) was recently developed, but it has not been as widely tested as the original E-PASS model (39). The number of included variables for the modified model is reduced from 10 to 7 . All the variables can be obtained before surgery using the mE-PASS model rather than the 3 extra variables (blood loss, operative time, and the extent of the skin incision) in the E-PASS model. The formula of PRS in the mE-PASS model is the same as in the E-PASS model, but the surgical stress score fixed (SSSf) was obtained directly according to the type of surgery. The modified E-PASS model was shown to be more convenient to implement, and it could assist surgeons in making perioperative decisions before the operation. However, the SSSf of the mE-PASS model has a crucial defect in that it was based on the Japanese population. Whether this system is suitable for patients from other countries remains unclear.

Several limitations of this study should be acknowledged: (I) seventeen studies were from Japan, and we had expected more studies about the E-PASS model from other countries, especially western countries; (II) only studies published in English were included in our study; (III) all the digestive surgeries that were included in our studies have the potential to cause high heterogeneity; (IV) and some data from Japan might have overlapped and introduced bias into our analysis.

\section{Conclusions}

In summary, the E-PASS model is a convenient and effective risk assessment tool for patients undergoing digestive surgeries, especially for the elderly. More stringently designed studies concerning this avenue of inquiry are expected to develop better estimates of the application value of this model in digestive surgeries, especially in western populations.

\section{Acknowledgments}

Funding: This work was supported by the National Key Research and Development Program (grant number 2016YFC0906400) and China's National Natural Science Foundation (grant number 81874059).

\section{Footnote}

Reporting Checklist: The authors have completed the PRISMA reporting checklist. Available at https://dx.doi. org/10.21037/apm-21-941

Conflicts of Interest: All authors have completed the ICMJE uniform disclosure form (available at https://dx.doi. org/10.21037/apm-21-941). The authors have no conflicts of interest to declare.

Ethical Statement: The authors are accountable for all aspects of the work in ensuring that questions related to the accuracy or integrity of any part of the work are appropriately investigated and resolved.

Open Access Statement: This is an Open Access article distributed in accordance with the Creative Commons Attribution-NonCommercial-NoDerivs 4.0 International License (CC BY-NC-ND 4.0), which permits the noncommercial replication and distribution of the article with the strict proviso that no changes or edits are made and the original work is properly cited (including links to both the formal publication through the relevant DOI and the license). See: https://creativecommons.org/licenses/by-nc-nd/4.0/.

\section{References}

1. Hashimoto D, Takamori H, Hirotaz M, et al. Prediction of operative morbidity after pancreatic resection. Hepatogastroenterology 2013;60:1577-82.

2. Loehrer AP, House MG, Nakeeb A, et al. Cholangiocarcinoma: are North American surgical outcomes optimal? J Am Coll Surg 2013;216:192-200.

3. Papenfuss WA, Kukar M, Oxenberg J, et al. Morbidity and mortality associated with gastrectomy for gastric cancer. Ann Surg Oncol 2014;21:3008-14.

4. Sano T, Sasako M, Yamamoto S, et al. Gastric cancer surgery: morbidity and mortality results from a prospective randomized controlled trial comparing D2 and extended para-aortic lymphadenectomy--Japan Clinical Oncology Group study 9501. J Clin Oncol 2004;22:2767-73.

5. Haga Y, Ikei S, Ogawa M. Estimation of Physiologic Ability and Surgical Stress (E-PASS) as a new prediction scoring system for postoperative morbidity and mortality following elective gastrointestinal surgery. Surg Today 1999;29:219-25.

6. Copeland GP, Jones D, Walters M. POSSUM: a scoring system for surgical audit. Br J Surg 1991;78:355-60.

7. Owens WD, Felts JA, Spitznagel EL Jr. ASA physical status classifications: a study of consistency of ratings. Anesthesiology 1978;49:239-43. 
8. Ariake K, Ueno T, Takahashi M, et al. E-PASS comprehensive risk score is a good predictor of postsurgical mortality from comorbid disease in elderly gastric cancer patients. J Surg Oncol 2014;109:586-92.

9. Ishino Y, Saigusa S, Ohi M, et al. Preoperative C-reactive protein and operative blood loss predict poor prognosis in patients with gastric cancer after laparoscopy-assisted gastrectomy. Asian J Endosc Surg 2014;7:287-94.

10. Haga Y, Miyamoto A, Wada Y, et al. Value of E-PASS models for predicting postoperative morbidity and mortality in resection of perihilar cholangiocarcinoma and gallbladder carcinoma. HPB (Oxford) 2016;18:271-8.

11. Tominaga T, Takeshita H, Takagi K, et al. E-PASS score as a useful predictor of postoperative complications and mortality after colorectal surgery in elderly patients. Int J Colorectal Dis 2016;31:217-25.

12. Kitano Y, Tsuji A, Inoue R, et al. E-PASS scoring system is a good predictor of prognosis in HCC patients underwent major hepatectomy. Hepatol Int 2016;10:S311.

13. Miyakita H, Sadahiro S, Saito G, et al. Risk scores as useful predictors of perioperative complications in patients with rectal cancer who received radical surgery. Int J Clin Oncol 2017;22:324-31.

14. Takahashi R, Nunobe S, Makuuchi R, et al. Survival outcomes of elderly patients with pathological stages II and III gastric cancer following curative gastrectomy. Ann Gastroenterol Surg 2020;4:433-40.

15. Yamamoto M, Saito H, Uejima C, et al. Estimation of Physiological Ability and Surgical Stress Score Is a Useful Prognostic Indicator for Elderly Patients with Colorectal Cancer. Dig Surg 2020;37:145-53.

16. Kondo H, Hirano Y, Ishii T, et al. E-PASS Scoring System May Be Useful for Prediction of Postoperative Complications in Super Elderly Colorectal Cancer Surgery Patients. J Anus Rectum Colon 2020;4:137-44.

17. Efanov M, Alikhanov R, Zamanov E, et al. Combining E-PASS model and disease specific risk factors to predict severe morbidity after liver and bile duct resection for perihilar cholangiocarcinoma. HPB (Oxford) 2021;23:387-93.

18. GA Wells BS, D O'Connell, J Peterson, et al. The Newcastle-Ottawa Scale (NOS) for assessing the quality of nonrandomised studies in meta-analyses. Available online: http://www.ohri.ca/programs/clinical_epidemiology/ oxford.asp

19. Abe H, Mafune K, Minamimura K, et al. Validation of the Estimation of Physiologic Ability and Surgical Stress (E-PASS) score for maintenance hemodialysis patients undergoing elective abdominal surgery. Dig Surg 2014;31:269-75.

20. Banz VM, Studer P, Inderbitzin D, et al. Validation of the estimation of physiologic ability and surgical stress (E-PASS) score in liver surgery. World J Surg 2009;33:1259-65.

21. Deyle S, Banz VM, Wagner M, et al. Estimation of physiologic ability and surgical stress score does not predict immediate outcome after pancreatic surgery. Pancreas 2011;40:723-9.

22. Hashimoto D, Takamori H, Sakamoto Y, et al. Is an estimation of physiologic ability and surgical stress able to predict operative morbidity after pancreaticoduodenectomy? J Hepatobiliary Pancreat Sci 2010;17:132-8.

23. Koushi K, Korenaga D, Kawanaka H, et al. Using the E-PASS scoring system to estimate the risk of emergency abdominal surgery in patients with acute gastrointestinal disease. Surg Today 2011;41:1481-5.

24. Hashimoto D, Takamori H, Sakamoto Y, et al. Can the physiologic ability and surgical stress (E-PASS) scoring system predict operative morbidity after distal pancreatectomy? Surg Today 2010;40:632-7.

25. Oka Y, Nishijima J, Oku K, et al. Usefulness of an estimation of physiologic ability and surgical stress (E-PASS) scoring system to predict the incidence of postoperative complications in gastrointestinal surgery. World J Surg 2005;29:1029-33.

26. Haga Y, Ikei S, Wada Y, et al. Evaluation of an Estimation of Physiologic Ability and Surgical Stress (E-PASS) scoring system to predict postoperative risk: a multicenter prospective study. Surg Today 2001;31:569-74.

27. Haga Y, Wada Y, Takeuchi H, et al. Estimation of physiologic ability and surgical stress (E-PASS) for a surgical audit in elective digestive surgery. Surgery 2004;135:586-94.

28. Hirose J, Mizuta H, Ide J, et al. E-PASS for predicting postoperative risk with hip fracture: a multicenter study. Clin Orthop Relat Res 2008;466:2833-41.

29. Haga Y, Ikejiri K, Takeuchi H, et al. Value of general surgical risk models for predicting postoperative liver failure and mortality following liver surgery. J Surg Oncol 2012;106:898-904.

30. Haga Y, Wada Y, Takeuchi H, et al. Prediction of anastomotic leak and its prognosis in digestive surgery. World J Surg 2011;35:716-22.

31. Kubota T, Hiki N, Nunobe S, et al. Significance of the inflammation-based Glasgow prognostic score for short- 
and long-term outcomes after curative resection of gastric cancer. J Gastrointest Surg 2012;16:2037-44.

32. Li MX, Liu XM, Zhang XF, et al. Prognostic role of neutrophil-to-lymphocyte ratio in colorectal cancer: a systematic review and meta-analysis. Int J Cancer 2014;134:2403-13.

33. Yang Y, Gao P, Chen X, et al. Prognostic significance of preoperative prognostic nutritional index in colorectal cancer: results from a retrospective cohort study and a meta-analysis. Oncotarget 2016;7:58543-52.

34. Man Z, Pang Q, Zhou L, et al. Prognostic significance of preoperative prognostic nutritional index in hepatocellular carcinoma: a meta-analysis. HPB (Oxford) 2018;20:888-95.

35. Hsueh SW, Liu KH, Hung CY, et al. Predicting Postoperative Events in Patients With Gastric Cancer: A Comparison of Five Nutrition Assessment Tools. In Vivo 2020;34:2803-9.

36. Kenig J, Mitus JW, Rapacz K, et al. Usefulness of

Cite this article as: Chen G, Chen Y, Dai Y, Shi Z, Pan J, Fan X, Lin H. The Estimation of Physiologic Ability and Surgical Stress (E-PASS) model as a predictor of postoperative complications and mortality after digestive surgeries: a metaanalysis and systematic review. Ann Palliat Med 2021;10(8):86728683. doi: 10.21037/apm-21-941 scoring systems in outcome prediction for older cancer patients undergoing abdominal surgery. Acta Chir Belg 2020;120:383-9.

37. Yamashita M, Adachi T, Ono S, et al. Evaluation of efficacy of physiologic ability and surgical stress score to predict postoperative complications and of long-term outcome in elderly patients with pancreaticoduodenectomy. HPB 2018;20:S644-5.

38. Chen T, Wang H, Wang H, et al. POSSUM and P-POSSUM as predictors of postoperative morbidity and mortality in patients undergoing hepato-biliarypancreatic surgery: a meta-analysis. Ann Surg Oncol 2013;20:2501-10.

39. Haga Y, Ikejiri K, Wada Y, et al. A multicenter prospective study of surgical audit systems. Ann Surg 2011;253:194-201.

(English Language Editors: J. Collie and J. Chapnick) 


\section{Supplementary}

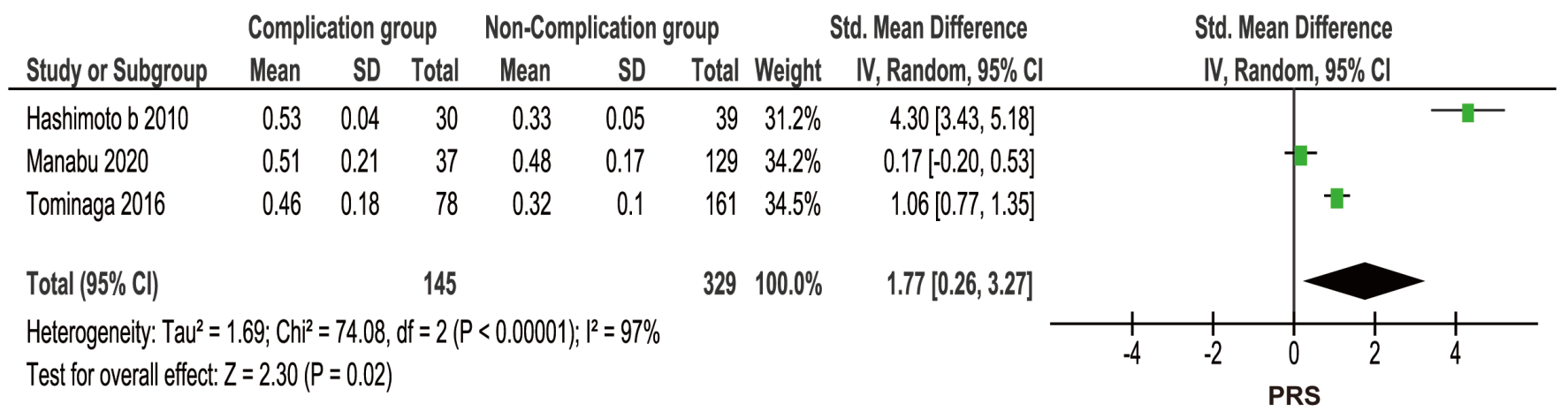

Figure S1 Forest plot of comparison: The group with postoperative complications group vs. The group without complications in the PRS. PRS, preoperative risk score. 
Table S1 summary of subgroup meta-analysis for PRS/SSS/CRS in patients with or without complications

\begin{tabular}{|c|c|c|c|c|c|c|c|c|}
\hline \multirow{2}{*}{ Subgroup } & \multirow{2}{*}{ Analysis } & \multirow{2}{*}{ Studies } & \multicolumn{2}{|c|}{ Random-effects model } & \multicolumn{2}{|c|}{ Fixed-effects model } & \multicolumn{2}{|c|}{ Heterogeneity } \\
\hline & & & OR $(95 \% \mathrm{Cl})$ & $\mathrm{P}$ & OR $(95 \% \mathrm{Cl})$ & $\mathrm{P}$ & $1^{2}$ & $\mathrm{Ph}$ \\
\hline \multicolumn{9}{|l|}{ PRS } \\
\hline \multirow[t]{2}{*}{ 1- Sample size } & $\geq 200$ & 3 & $0.37(-0.28,1.02)$ & 0.267 & $0.32(0.17,0.51)$ & $<0.001$ & $94 \%$ & $<0.001$ \\
\hline & $<200$ & 3 & $1.46(-0.47,3.39)$ & 0.139 & $0.52(0.25,0.80)$ & $<0.001$ & $98 \%$ & $<0.001$ \\
\hline 2- Location & Not Japan & 2 & $0.03(-0.15,0.21)$ & 0.750 & $0.03(-0.15,0.21)$ & 0.750 & 0 & 0.778 \\
\hline \multirow[t]{2}{*}{ 3- Surgery } & Gl surgery & 3 & $0.43(-0.27,1.13)$ & 0.232 & $0.59(0.39,0.79)$ & $<0.001$ & $91 \%$ & $<0.001$ \\
\hline & HBP surgery & 3 & $1.35(0.00,2.70)$ & 0.050 & $0.21(0.03,0.38)$ & 0.023 & $98 \%$ & $<0.001$ \\
\hline 4- Age & Elderly & 2 & $0.62(-0.26,1.50)$ & 0.165 & $0.72(0.50,0.95)$ & $<0.001$ & $93 \%$ & $<0.001$ \\
\hline 5- CRS cut-off & Not 0.5 & 3 & $1.79(0.26,3.31)$ & 0.022 & $0.95(0.73,1.17)$ & $<0.001$ & $97 \%$ & $<0.001$ \\
\hline \multicolumn{9}{|l|}{ SSS } \\
\hline \multirow[t]{2}{*}{ 1- Sample size } & $\geq 200$ & 3 & $0.33(0.17,0.50)$ & $<0.001$ & $0.33(0.18,0.48)$ & $<0.001$ & $16 \%$ & 0.306 \\
\hline & $<200$ & 3 & $1.12(0.10,2.15)$ & 0.031 & $0.86(0.60,1.13)$ & $<0.001$ & $93 \%$ & $<0.001$ \\
\hline \multirow[t]{2}{*}{ 2- Location } & Japan & 4 & $0.94(0.29,1.60)$ & 0.005 & $0.69(0.50,0.88)$ & $<0.001$ & $90 \%$ & $<0.001$ \\
\hline & Not Japan & 2 & $0.25(0.07,0.44)$ & 0.006 & $0.25(0.07,0.44)$ & 0.006 & 0 & 0.798 \\
\hline \multirow[t]{2}{*}{ 3- Surgery } & Gl surgery & 3 & $0.52(0.32,0.72)$ & $<0.001$ & $0.52(0.32,0.72)$ & $<0.001$ & 0 & 0.828 \\
\hline & HBP surgery & 3 & $0.89(0.03,1.75)$ & 0.043 & $0.42(0.25,0.59)$ & $<0.001$ & $95 \%$ & $<0.001$ \\
\hline \multirow[t]{2}{*}{ 1- Sample size } & $\geq 200$ & 3 & $0.47(-0.01,1.51)$ & 0.056 & $0.44(0.29,0.59)$ & $<0.001$ & $90 \%$ & $<0.001$ \\
\hline & $<200$ & 5 & $1.41(0.43,2.40)$ & 0.005 & $0.94(0.71,1.17)$ & $<0.001$ & $94 \%$ & $<0.001$ \\
\hline \multirow[t]{2}{*}{ 2- Location } & Japan & 6 & $1.31(0.61,2.01)$ & $<0.001$ & $0.96(0.78,1.14)$ & $<0.001$ & $93 \%$ & $<0.001$ \\
\hline & Not Japan & 2 & $0.22(0.04,1.51)$ & 0.019 & $0.22(0.04,0.40)$ & 0.019 & 0 & 0.626 \\
\hline \multirow[t]{2}{*}{ 3- Surgery } & Gl surgery & 4 & $0.74(0.38,1.10)$ & $<0.001$ & $0.77(0.57,0.96)$ & $<0.001$ & $66 \%$ & 0.033 \\
\hline & HBP surgery & 4 & $1.33(0.31,2.34)$ & 0.010 & $0.46(0.29,0.63)$ & $<0.001$ & $97 \%$ & $<0.001$ \\
\hline \multirow[t]{2}{*}{ 4- Age } & Elderly & 2 & $0.76(0.29,1.23)$ & 0.002 & $0.80(0.58,1.03)$ & $<0.001$ & $76 \%$ & 0.043 \\
\hline & Younger & 6 & $1.13(0.40,1.85)$ & 0.002 & $0.49(0.34,0.65)$ & $<0.001$ & $94 \%$ & $<0.001$ \\
\hline \multirow[t]{2}{*}{ 5- CRS cut-off } & 0.5 & 5 & $0.55(0.19,0.91)$ & 0.009 & $0.37(0.21,0.52)$ & $<0.001$ & $76 \%$ & 0.002 \\
\hline & Not 0.5 & 3 & $1.78(0.44,3.12)$ & 0.003 & $1.02(0.81,1.24)$ & $<0.001$ & $97 \%$ & $<0.001$ \\
\hline
\end{tabular}

OR: odds ratio; Cl: confidence interval; Ph: $\mathrm{P}$ value of $\mathrm{Q}$ test for heterogeneity test; Gl: gastrointestinal; HBP: hepato-biliary-pancreatic; PRS: preoperative risk score; SSS: surgical stress score; CRS: comprehensive risk score. 

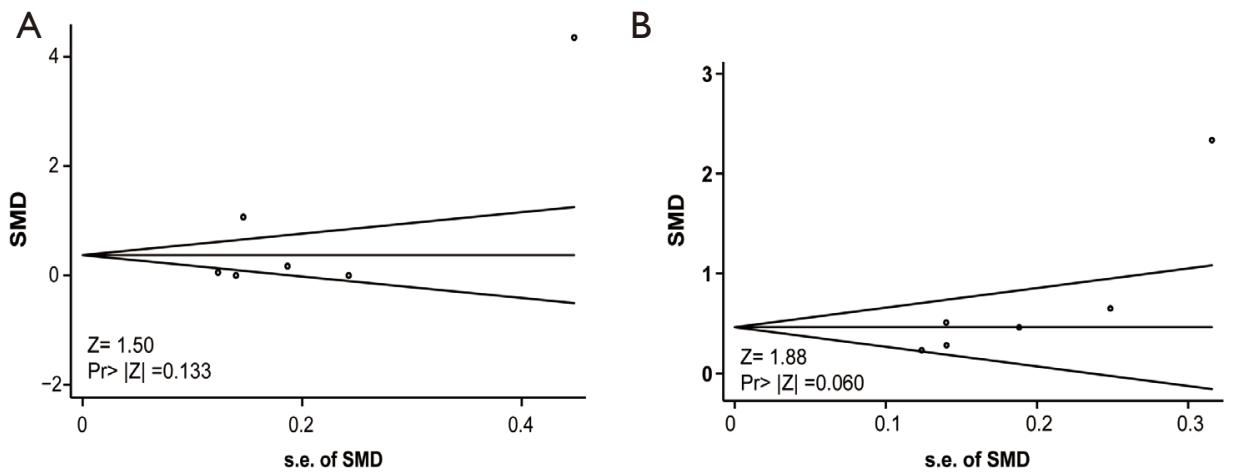

C

D
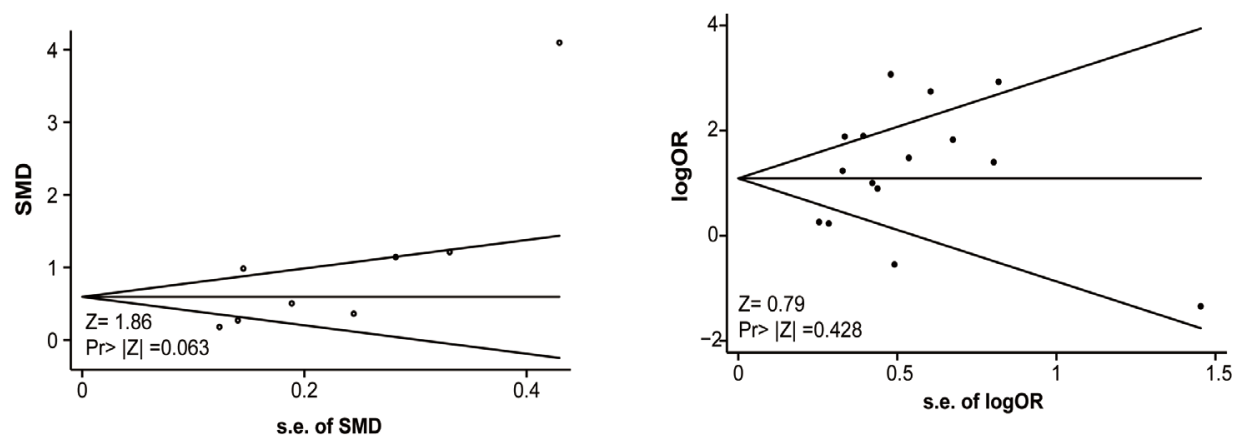

E

$\mathrm{F}$
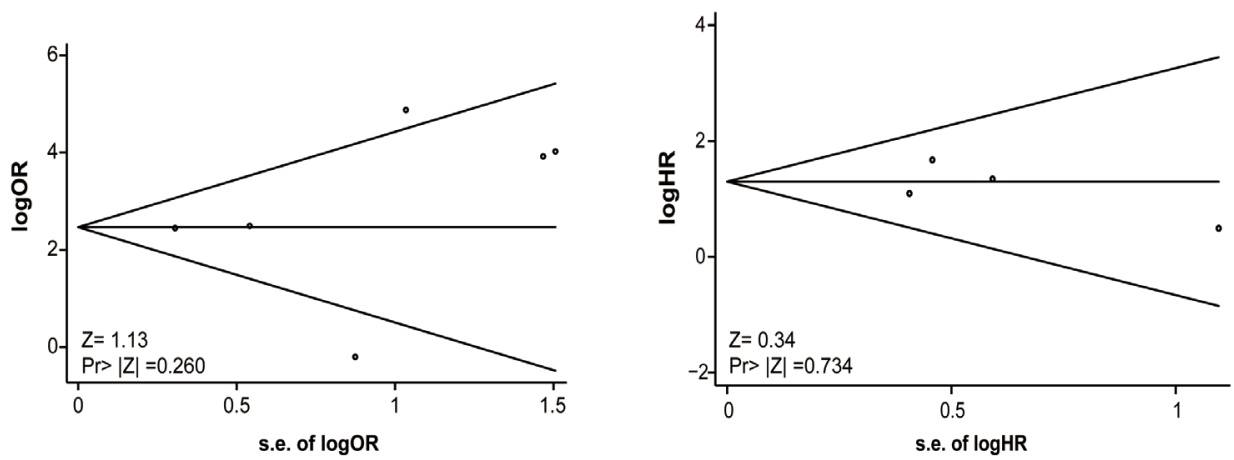

Figure S2 Funnel plots of publication bias for pooled analyses using Begg's test. (A) The PRS; (B) The SSS; (C) The CRS; (D) postoperative morbidity; (E) mortality; (F) overall survival. PRS: preoperative risk score; SSS: surgical stress score; CRS: comprehensive risk score. 